\title{
Latex Agglutination Turbidimetric Immunoassay Versus Enzyme-Linked Immunosorbent Assay for Helicobacter pylori: An Observational Study.
}

\section{Yoshitaka Tokai ( $\nabla$ yoshitaka.tokai@jfcr.or.jp )}

The Cancer Institute Hospital

Junko Fujisaki

The Cancer Institute Hospital

Naoki Ishizuka

The Cancer Institute Hospital

Hiroki Osumi

The Cancer Institute Hospital

Ken Namikawa

The Cancer Institute Hospital

Shoichi Yoshimizu

The Cancer Institute Hospital

\section{Yusuke Horiuchi}

The Cancer Institute Hospital

Akiyoshi Ishiyama

The Cancer Institute Hospital

Toshiyuki Yoshio

The Cancer Institute Hospital

Toshiaki Hirasawa

The Cancer Institute Hospital

Kazumasa Miki

The Cancer Institute Hospital

\section{Tomohiro tsuchida}

The Cancer Institute Hospital

\section{Research Article}

Keywords: Helicobacter pylori, Enzyme-Linked Immunosorbent Assay, Latex Agglutination Tests

Posted Date: December 10th, 2020

DOl: https://doi.org/10.21203/rs.3.rs-120423/v1 
License: (c) (i) This work is licensed under a Creative Commons Attribution 4.0 International License. Read Full License 


\section{Abstract}

Background: Helicobacter pylori antibody levels in the blood are currently measured using an enzyme-linked immunosorbent assay (ELISA). In April 2016, FUJIFILM Wako Pure Chemical Corporation launched the "Ltype Wako Helicobacter pylori antibody J" test, which is based on the latex agglutination turbidimetric immunoassay. In this study, we investigated the usefulness of the Wako test.

Methods: We measured H. pylori antibody levels using both ELISA and Wako tests in 180 patients who underwent esophagogastroduodenoscopy at our hospital between September 2017 and February 2019. Ninety patients had $H$. pylori infections. We calculated the diagnostic accuracy, sensitivity, and specificity of each test and the concordance rate between the two tests. If the lower limits of $90 \%$ confidence intervals (Cl) for each diagnostic validity exceeded the $85 \%$ threshold, the usefulness of the diagnostic test was confirmed.

Results: Diagnostic accuracy, sensitivity, and specificity were $94.4 \%(90 \% \mathrm{Cl} ; 90.8-97.0 \%), 94.4 \%$ (90\% Cl; 88.7-97.8\%), and $94.4 \%$ (90\% Cl; 88.7-97.8\%), respectively, using the Wako test, and $94.4 \%$ (90\% Cl; $90.8-$ $97.0 \%), 88.9 \%$ (90\% Cl; $81.9-93.8 \%)$, and $100 \%$ (90\% Cl; $96.0-100 \%)$, respectively, using ELISA. The concordance rate between the two tests was high $(\mathrm{K}=0.8444)$.

Conclusions: We confirmed the usefulness of the Wako test, especially when screening for $H$. pylori infection, due to its high sensitivity.

Trial registration: We retrospectively registered the data of this study.

\section{Background}

Helicobacter pylori, a gram-negative bacillus, was discovered in 1983 by Warren and Marshall and is known to persistently infect the human gastric mucosa and induce histological gastritis accompanied by neutrophilic infiltration [1]. It has been demonstrated that $H$. pylori infection is associated with the development of gastric ulcer/duodenal ulcers and gastric cancer [2]. In 1994, the International Agency for Research on Cancer, an external organisation of the World Health Organisation, reported that $H$. pylori is a cause of gastric cancer [3].

People in Japan were notified that eradication therapy for $H$. pylori-infected gastritis would be covered by health insurance starting February 2013; thus, the diagnosis of $H$. pylori infection became more prevalent.

Diagnostic methods for $H$. pylori infection with endoscopy include the culture test, microscopic test, and rapid urease test. The tests without endoscopy include the urea breath test (UBT), blood $H$. pylori antibody test, and faeces $\mathrm{H}$. pylori antigen test [4, 5]. According to the Japanese Society for Helicobacter Research guidelines [6], UBT is the most accurate among the tests, and its sensitivity and specificity are $98 \%$ [7].

The blood H. pylori antibody test is convenient and widely used to diagnose $H$. pylori infection in current clinical practice. The prevailing methods for this antibody test are the enzyme-linked immunosorbent assay 
(ELISA, Eiken Chemical, Tokyo, Japan) and the chemiluminescent method, both of which have limitations: analysis is time-consuming and requires special measuring devices.

In April 2016, FUJIFILM Wako Pure Chemical Corporation launched the "L-type Wako H. pylori antibody J" $(\mathrm{L}-\mathrm{HP} \cdot \mathrm{J})$ test (FUJIFILM Wako Pure Chemical Corporation, Osaka, Japan), which is based on the latex agglutination turbidimetric immunoassay.

Since this kit can be used with a general-purpose automatic analyser, it is less complicated than ELISA. Many samples can be analysed simultaneously, rapidly, and more economically using this kit. Another advantage of this kit is that the presence of $H$. pylori infection can be diagnosed, and drugs can be prescribed to eradicate $H$. pylori on the same day as the blood test. Consequently, this kit might contribute to reduced medical costs; however, its accuracy has not been confirmed. In this study, we evaluated the diagnostic validity of the L-HP.J test, which has not yet been demonstrated, by calculating and comparing its diagnostic accuracy, sensitivity, specificity, and concordance rate with those of the standard ELISA method.

\section{Methods}

\section{Patients}

This observational study was conducted in clinical settings and included consecutive patients who underwent gastrointestinal endoscopy between September 2017 and February 2019. The following patients were excluded: those who had received eradication therapy for $H$. pylori or had undergone gastrectomy; those who had taken proton pump inhibitors within 2 weeks before undergoing the UBT; those who had taken antibiotics within 4 weeks before the endoscopy; those who had undergone head and neck surgery and were unable to undergo the UBT; those in whom advanced gastric cancer was detected endoscopically; and those who were suspected of having type A gastritis endoscopically (cases with severe atrophic gastritis of the corpus and mild or no atrophic gastritis of the antrum). Our medical staff interviewed the patients to determine whether they had received eradication therapy for $H$. pylori or had undergone gastrectomy in the past. Major reasons for undergoing gastrointestinal endoscopy included screening, testing positive for $\mathrm{H}$. pylori antibodies, having abdominal symptoms such as upper abdominal pain, and workup for early gastric cancer. Ultimately, 180 patients were enrolled in the study.

\section{Serum H. pylori kits and assay procedure}

We measured $H$. pylori antibodies in-house, in specimens from subjects who provided consent, using (1) the L-HP.J test and (2) the E-plate "Eiken" H. pylori antibody test (ELISA). Blood sampling was performed on the same day as endoscopy. Cut-off values used for these antibody tests were as follows: (1) L-HP.J test, 4 $\mathrm{U} / \mathrm{mL}$ or more was considered positive for $H$. pylori, and (2) ELISA, $10 \mathrm{U} / \mathrm{mL}$ or more was considered positive for $H$. pylori. Measurement using each kit was performed in-house. In this study, we used a full automicroplate chemiluminescence analyser (Hitachi Chemical Diagnostics Systems Co., Ltd., Tokyo, Japan) for ELISA, and Hitachi Labospect 008 automatic biochemistry analyser (Hitachi HighTechnologies,Tokyo, Japan) for the L-HP.J test. The ELISA consists of a combination of manual (by hand) 
and automated (by auto) steps (Fig. 1). First, the specimen and buffer are manually dispensed into the wells of a 96-well microplate. This step may require a few minutes per sample, as processing speed is dependent on the technician's skill level. An automated washing step is then performed to remove any unbound antibodies. A number of additional incubation and washing steps are performed before the automated analysis of the microplate. The entire process takes approximately 87 minutes to analyse all 96 specimens. In contrast, the latex agglutination turbidimetric immunoassay does not require the incubation and washing steps that the ELISA does; hence, one specimen can be analysed in 10 minutes, and the results of 100 specimens can be obtained in 16 min. Reagents were provided by the FUJIFILM Wako Pure Chemical Corporation. Fresh plasma was used for analysis via ELISA, and cryopreserved sera $\left(-20^{\circ} \mathrm{C}\right.$; harvested within 4 weeks) or fresh plasma were thawed and used for analysis via the L-HP.J test.

\section{Endoscopic findings}

Esophagogastroduodenoscopy (EGD) was performed with the Evis Lucera Elite system (Olympus Corporation, Tokyo, Japan) and a GIF-H290Z scope (Olympus Corporation). EGD was performed by endoscopists who have 5000 cases or more of experience in EGD. The grading of gastric mucosal atrophy by endoscopic findings was based on the Kimura-Takemoto classification [8]: no atrophy (C-0); closed-type (C-1 to $\mathrm{C}-3$ ), in which an endoscopic atrophic border is in the lesser curvature of the gastric body and does not extend beyond the cardia; and open-type (0-1 to 0-3), in which an endoscopic atrophic border extends beyond the cardia and progresses into the greater curvature.

Findings that suggest active gastritis, such as spotty or diffuse redness from the gastric body to the fundus, accompanied by disappearance of the regular arrangement of collecting venules, enlarged/tortuous folds, mucosal swelling, and sticky mucus according to the Kyoto classification of gastritis [9], were considered as presence of $H$. pylori infection (Fig. 2).

\section{Criteria for determining H. pylori infection status and methods to evaluate the kits}

H. pylori infection was diagnosed by integrating the endoscopic findings and the UBT results as follows: Subjects who had no gastric mucosal atrophy or any findings suggestive of active gastritis on EGD as described above, and whose UBT results were negative ( $\geq 2.5 \%$ o was defined as positive) (UBIT, Otsuka Pharmaceutical), were considered uninfected, while those who had findings of either gastric mucosal atrophy or active gastritis detected by EGD with a positive UBT result were considered as infected. Patients whose endoscopic findings and UBT results had a discrepancy, such as active gastritis detected endoscopically, indicating a current $H$. pylori infection, but negative UBT results, and conversely, patients who were not infected according to their endoscopic findings but whose UBT results were positive, were excluded from the study.

Subjects whose endoscopic findings and UBT results concurred in terms of the presence/absence of $H$. pylori infection were diagnosed as being positive or negative for $H$. pylori, and the sensitivity, specificity, and diagnostic accuracy of the L-HP•J test were determined. ELISA was performed in a similar manner, and the 
concordance rate with the L-HP.J test was calculated. In cases misdiagnosed by the L-HP•J test, the test results correlated with the clinical findings.

\section{Sample size calculation}

According to a previous report from Japan, the sensitivity and specificity of the L-HP.J test for $H$. pylori positive cases were $96.4 \%$ (currently infected; 56 cases) and 97.7\% (uninfected; 86 cases), respectively. Therefore, we set the expected value as $95 \%$, and threshold value as $85 \%$ for both sensitivity and specificity. Based on the binomial test, a sample size of 85 cases was deemed to be required for both uninfected and currently infected cases using the following criteria: one-sided alpha of $5 \%$ and power of $90 \%$. Ultimately, a total of 180 patients (90 uninfected and 90 currently infected cases) were enrolled to compensate for any dropouts.

\section{Statistical analyses}

All continuous variables are expressed as the median and range. For diagnostic performance, diagnostic accuracy, sensitivity, and specificity are presented as percentages with a confidence interval (CI) of $90 \%$. To evaluate concordance between the test methods for positivity and negativity, the $\mathrm{k}$ statistic was used. McNemar's test, which evaluates marginal homogeneity between two test methods, was used to compare the L-HP.J test and ELISA. A P-value $<0.05$ was considered statistically significant. Statistical analysis was performed using Statistical Analysis System (SAS) software 9.4 (TS1M5, SAS Institute Inc., Cary, NC, USA).

\section{Ethics}

This study was approved by the Institutional Review Board of Cancer Institute Hospital (No. 2016 - 1208), and performed in compliance with the principles of the Declaration of Helsinki and its later amendments. While recording the data for this study, all personal identifying information was removed. Informed consent for the use of pathological specimens and imaging data for research purposes was obtained from each patient.

\section{Results}

\section{Breakdown of $\mathrm{H}$. pylori infection status}

Among the 180 patients, 90 patients (50\%) were uninfected and 90 patients (50\%) had a H. pylori infection. Regarding the patient characteristics of uninfected and currently infected cases, the median age and percentage of male patients was 57 years (range; 37-76), and 65 years (range; 28-87), 45.6\% and 57.8\%, respectively. The uninfected group was younger in age. Among the currently infected subjects, the degree of gastric mucosal atrophy (the Kimura-Takemoto classification) was C-1 to C-3 in 51.1\% (46/90) and 0-1 to 0-3 in 48.9\% (44/90). Although the major comorbidity in the currently infected subjects was early gastric cancer, this was seen only in one patient among the uninfected subjects (Table 1). 
Table 1

Characteristics of subjects with and without a current Helicobacter pylori infection.

\begin{tabular}{|c|c|c|c|}
\hline & $\begin{array}{l}\text { Uninfected } \\
n=90\end{array}$ & $\begin{array}{l}\text { Currently infected } \\
n=90\end{array}$ & $P$-value \\
\hline Age, median (range) & $57(37-76)$ & $65(28-87)$ & $<0.001$ \\
\hline Sex, n (\%) & $41(45.6 \%)$ & $52(57.8 \%)$ & 0.14 \\
\hline Male & $49(54.4 \%)$ & $38(42.2 \%)$ & \\
\hline \multicolumn{4}{|l|}{ Female } \\
\hline Degree of atrophy, n (\%) & $90(100 \%)$ & $0(0 \%)$ & $<0.001$ \\
\hline No atrophy & $0(0 \%)$ & $46(51.1 \%)$ & \\
\hline Closed-type atrophy & $0(0 \%)$ & $44(48.9 \%)$ & \\
\hline \multicolumn{4}{|l|}{ Open-type atrophy } \\
\hline Titre of L-HP.J test $(\mathrm{U} / \mathrm{mL})$, median (range) & $0.75(0-13.6)$ & $32.7(2.7-109.8)$ & $<0.001$ \\
\hline UBT (\%), median (range) & $0.4(0-1.6)$ & $13.8(2.7-135.3)$ & $<0.001$ \\
\hline Comorbidity & $1(1.1 \%)$ & $28(31.1 \%)$ & $<0.001$ \\
\hline Early gastric cancer, n (\%) & $1(1.1 \%)$ & $5(5.6 \%)$ & 0.21 \\
\hline Superficial oesophageal cancer, n (\%) & $1(1.1 \%)$ & $0(0 \%)$ & 1 \\
\hline Duodenal cancer, n (\%) & $0(0 \%)$ & $2(2.2 \%)$ & 0.49 \\
\hline Post ESD for early gastric cancer, $\mathrm{n}(\%)$ & $0(0 \%)$ & $1(1.1 \%)$ & 1 \\
\hline Post ESD for superficial oesophageal cancer, $\mathrm{n}(\%)$ & $0(0 \%)$ & $1(1.1 \%)$ & 1 \\
\hline Gastric ulcer scar, n (\%) & & & \\
\hline
\end{tabular}

\section{Diagnostic validity of the L-HP•J test (diagnostic accuracy, sensitivity, and specificity)}

The diagnostic accuracy, sensitivity, and specificity for current $H$. pylori infection were $94.4 \%(90 \% \mathrm{Cl}$; $90.8-$ 97.0\%), 94.4\% (90\% Cl; 88.7-97.8\%), and 94.4\% (90\% Cl; 88.7-97.8\%), respectively, when the L-HP.J test was used, and $94.4 \%$ (90\% Cl; $90.8-97.0 \%), 88.9 \%$ (90\% Cl; $81.9-93.8 \%$ ), and $100 \%$ (90\% Cl; $96.0-100 \%)$, respectively, when ELISA was used (Table 2). Although the lower limits of $90 \% \mathrm{Cl}$ of the L-HP.J test exceeded the threshold value (85\%) in terms of diagnostic accuracy, sensitivity, and specificity, the lower limits of $90 \% \mathrm{Cl}$ for sensitivity of ELISA (81.9\%) were lower than the threshold value (85\%). 
Table 2

Diagnostic validity of the L-type Wako Helicobacter pylori antibody $\mathrm{J}$ test and the Enzyme-Linked Immunosorbent Assay.

\begin{tabular}{|lll|}
\hline & L-HP.J test & ELISA \\
\hline Diagnostic accuracy, \% & $94.4 \%$ & $94.4 \%$ \\
$(90 \% \mathrm{Cl})$ & $(90.8-97.0 \%)$ & $(90.8-97.0 \%)$ \\
\hline Sensitivity, \% & $94.4 \%$ & $88.9 \%$ \\
$(90 \% \mathrm{Cl})$ & $(88.7-97.8 \%)$ & $(81.9-93.8 \%)$ \\
\hline Specificity, \% & $94.4 \%$ & $100 \%$ \\
$(90 \% \mathrm{Cl})$ & $(88.7-97.8 \%)$ & $(96.0-100 \%)$ \\
\hline ELISA, Enzyme-Linked Immunosorbent Assay; L-HP.J, L-type Wako Helicobacter pylori antibody J; $90 \%$ \\
\hline Cl, 90\% Confidence Interval.
\end{tabular}

\section{Comparison of concordance rates with ELISA (conventional test)}

The number of subjects who were positive for anti-H. pylori antibody using the ELISA and L-HP.J test were 80 and 90 , respectively. The concordance rates between the L-HP.J test and ELISA were high $(\mathrm{k}=0.8444)$ (Table 3). Subsequently, using McNemar's test, we analysed the tendency for positive or negative results for $H$. pylori between the L-HP.J test and ELISA. The results revealed that ELISA had a significant tendency to evaluate the test cases as negative $(P=0.0129)$ (Table 3$)$.

Table 3

Investigation of concordance rates between the L-type Wako Helicobacter pylori antibody $\mathrm{J}$ test and the Enzyme-Linked Immunosorbent Assay.

\begin{tabular}{|c|c|c|c|c|c|}
\hline & $\begin{array}{l}\text { ELISA } \\
\text { Positive }\end{array}$ & $\begin{array}{l}\text { ELISA } \\
\text { Negative }\end{array}$ & Total & $P$-value & K \\
\hline $\begin{array}{l}\text { L-HP·J test } \\
\text { Positive }\end{array}$ & 78 & 12 & 90 & 0.0129 & $\begin{array}{l}0.844 \\
(0.767-0.922)\end{array}$ \\
\hline $\begin{array}{l}\text { L-HP.J test } \\
\text { Negative }\end{array}$ & 2 & 88 & 90 & & \\
\hline Total & 80 & 100 & 180 & & \\
\hline$P$-values we & mined via & mar's test. & & & \\
\hline
\end{tabular}

Investigation of cases misdiagnosed by the L-HP·J test 
Cases misdiagnosed by the L-HP.J test included five false negative cases (5.6\%) and five false positive cases (5.6\%) (Table 4). Among the false positive cases, one subject had a high titre, while the other four subjects had titres slightly above the cut-off value. All false negative subjects had open-type mucosal atrophy. 
Table 4

Misdiagnosed cases by the L-type Wako Helicobacter pylori antibody J test.

H. pylori antibody measurement result

Urea breath test

Endoscopic findings

\begin{tabular}{|c|c|c|c|c|c|c|c|c|c|}
\hline $\begin{array}{l}\text { Case } \\
\text { number }\end{array}$ & Sex & Age & $\begin{array}{l}\mathrm{L}- \\
\mathrm{HP} \cdot J\end{array}$ & $\begin{array}{l}\text { Judge- } \\
\text { ment }\end{array}$ & $\begin{array}{l}\text { ELISA } \\
(\mathrm{U} / \mathrm{ml})\end{array}$ & $\begin{array}{l}\text { Judge- } \\
\text { ment }\end{array}$ & $\begin{array}{l}\text { Change } \\
\text { rate } \\
(\% \text { o) }\end{array}$ & Judgment & $\begin{array}{l}\text { Kimura- } \\
\text { Takemoto } \\
\text { classification }\end{array}$ \\
\hline
\end{tabular}

False positive cases of L-HP·J

$\begin{array}{llllllllll}1 & \text { M } & 68 & 4.1 & + & 3.3 & - & 0.5 & - & \text { No atrophy } \\ 2 & \text { F } & 63 & 4.3 & + & 3 & - & 0.6 & - & \text { No atrophy } \\ 3 & \text { M } & 72 & 5.2 & + & 3 & - & 0.5 & - & \text { No atrophy } \\ 4 & \text { F } & 54 & 5.2 & + & 5.5 & - & 0.0 & - & \text { No atrophy } \\ 5 & \text { M } & 73 & 13.6 & + & 4.7 & - & 0.2 & - & \text { No atrophy }\end{array}$

False negative cases of L-HP.J

$\begin{array}{llllllllll}6 & F & 63 & 3.9 & - & 14 & + & 3.3 & + & 0-3 \\ 7 & \text { F } & 76 & 3.1 & - & 15.5 & + & 6 & + & 0-3 \\ 8 & \text { F } & 69 & 3.5 & - & 4.8 & - & 9.5 & + & 0-3 \\ 9 & \text { M } & 84 & 2.7 & - & 7.5 & - & 59.2 & + & 0-1 \\ 10 & \text { F } & 67 & 3.24 & - & 3 & - & 2.7 & + & 0-2\end{array}$

False negative cases of ELISA

\begin{tabular}{|llllllllll|}
1 & $\mathrm{M}$ & 28 & 16.56 & + & 6.4 & - & 5.7 & + & $0-1$ \\
\hline 2 & $\mathrm{M}$ & 57 & 7.2 & + & 9.7 & - & 13.9 & + & $\mathrm{C}-2$ \\
3 & $\mathrm{~F}$ & 69 & 3.5 & - & 4.8 & - & 3.7 & + & $0-3$ \\
4 & $\mathrm{M}$ & 70 & 5.9 & + & 5.9 & - & 3.9 & + & $0-1$ \\
\hline 5 & $\mathrm{~F}$ & 39 & 16.1 & + & 6.9 & - & 5 & + & $\mathrm{C}-1$ \\
\hline 6 & $\mathrm{M}$ & 84 & 2.7 & - & 7.5 & - & 59.2 & + & $0-1$ \\
7 & $\mathrm{~F}$ & 67 & 3.24 & - & 3 & - & 2.7 & + & $0-2$ \\
8 & $\mathrm{M}$ & 56 & 18.2 & + & 3 & - & 6.8 & + & $0-1$ \\
\hline 9 & $\mathrm{M}$ & 66 & 5.5 & + & 5.5 & - & 5.1 & + & $0-1$ \\
\hline 10 & $\mathrm{M}$ & 70 & 18.6 & + & 8.4 & - & 24.1 & + & $0-2$ \\
\hline
\end{tabular}




\section{Discussion}

Invasive diagnostic methods for $\mathrm{H}$. pylori infection listed in the Japanese Society for Helicobacter Research guidelines [6] include the rapid urease test, microscopic test, and culture test. Non-invasive methods include the UBT, blood H. pylori antibody test, and faeces $H$. pylori antigen test. Among them, the blood $H$. pylori antibody test is relatively convenient and widely used. A prevailing method for measuring $H$. pylori antibodies is ELISA.

In this study, we investigated a newly launched kit that can be used with a general-purpose automatic analyser: The L-HP.J test kit. The L-HP.J test can be performed using commercially available automated analysers, such as Hitachi 7180 (Hitachi High-Tech), JCA-BM6050 (JEOL Ltd., Tokyo, Japan), ARCHITECT c16000 (Canon Medical Systems, Tokyo, Japan), and DxC700AU (Beckman Coulter K.K., Tokyo, Japan). The L-HP.J test was launched in April 2016 by FUJIFILM Wako Pure Chemical Corporation and is based on the latex agglutination turbidimetric immunoassay. To our knowledge, this is the first report to evaluate the diagnostic validity of this newly launched kit. We demonstrated that the diagnostic accuracy, sensitivity, and specificity of the L-HP.J test was approximately $95 \%$, and the concordance rate between the L-HP.J test and conventional ELISA was high, with a $\mathrm{k}$ statistic of 0.8444 . Although ELISA showed high specificity, the $90 \% \mathrm{Cl}$ lower limits for sensitivity of ELISA did not exceed the threshold value of $85 \%$. In addition to this, ELISA had a significant tendency to give false negative results $(P=0.0129)$. From these findings, it can be considered that ELISA tends to underestimate the antibody titre, and that the L-HP.J test is a relatively more accurate kit.

The latex agglutination turbidimetric immunoassay is superior to ELISA in terms of throughput and reducing labour and is expected to greatly contribute to rapid testing. Kita et al. demonstrated that the diagnosis of $H$. pylori infection is improved when two strains from genotypes commonly seen in the Japanese population (\#3 and \#5) [10] are used. The L-HP.J test we evaluated in this study used the same two antigens as the chemiluminescent test. Our findings show that the accuracy of the L-HP.J test is not inferior to ELISA and that it has a greater sensitivity. Regarding the false positive and false negative cases detected using the L-HP.J test, all but one false positive case had low-positive titres (between $4 \mathrm{U} / \mathrm{mL}$ and 8.7 $\mathrm{U} / \mathrm{mL}$ ). It has been reported that approximately $10 \%$ of individuals with low-positive titres detected using the L-HP.J test are uninfected [11]. Thus, we believe that this problem arises due to the established cut-off value. Regarding the false negative cases, there were many subjects with open-type mucosal atrophy. This is because, in patients with severe atrophic gastritis, H. pylori colonisation is decreased or has spontaneously disappeared, resulting in the decrease of serum antibody [12]. It is important to use additional tests, such as the UBT, to diagnose $H$. pylori infection correctly if the L-HP.J test is not consistent with endoscopic findings, such as in the following two scenarios: (1) when an individual has an open-type mucosal atrophy and endoscopic findings suggest current infection, but the L-HP.J test result is negative; or (2) when endoscopic findings suggest no infection, but the L-HP.J test result has low-positive titres (between $4 \mathrm{U} / \mathrm{mL}$ and $8.7 \mathrm{U} / \mathrm{mL}$ ).

In the present study, we considered both the results of the UBT, which has a high diagnostic accuracy [13], and endoscopic findings based on the Kyoto classification of gastritis. We then diagnosed the presence or 
absence of $H$. pylori infection in those subjects whose UBT results and endoscopic findings were consistent. Therefore, subjects who were previously infected with $\mathrm{H}$. pylori and those who had type A gastritis could be excluded from the study. Moreover, this was a prospective study and antibody measurements using both kits were performed in-house, which increases the reliability of the study.

There are some limitations of the study. First, it represented a single-centre observational study. To confirm the generality of this kit, a multi-centre study is needed in the future. Second, in some subjects, fresh plasma was used for detection using ELISA and part of the L-HP.J test, while stored serum (harvested within 4 weeks) was also used for some of the L-HP.J tests; therefore, there were differences between subjects in terms of the conditions of the specimens used. However, supplemental data from FUJIFILM Wako Pure Chemical Corporation showed no difference in the titres between fresh plasma and stored serum (harvested within 4 weeks) under conditions of $-20^{\circ} \mathrm{C}$. Third, we used the UBT as the gold standard for diagnosing $H$ pylori infection; however, its accuracy is not $100 \%$ [13]. Fourth, we investigated only noninfected and currently infected cases, not previously infected cases. Therefore, a further study should be conducted to analyse the association between previously infected cases and the antibody titre of the LHP.J test.

\section{Conclusions}

The L-HP.J test has a high diagnostic performance and is not inferior to ELISA. It is useful, especially in screening for Helicobacter-pylori infection, due to its high sensitivity. It can be used with various generalpurpose automatic analysers because the kit is versatile, easy to use, and convenient. It can, therefore, contribute to the streamlining of clinical laboratory procedures.

\section{Abbreviations}

Confidence interval (Cl); enzyme-linked immunosorbent assay (ELISA); Esophagogastroduodenoscopy (EGD); L-type Wako H. pylori antibody J (L-HP.J); Urea breath test (UBT).

\section{Declarations}

\section{Ethics approval and consent to participate}

All procedures followed were in accordance with the ethical standards of the responsible committee on human experimentation (institutional and national) and with the Helsinki Declaration of 1964 and later versions. Informed consent Informed consent to be included in the study, or the equivalent, was obtained from all patients.

\section{Consent for publication}

Consent for publication has been obtained from patients.

\section{Availability of data and materials}


The datasets used and/or analysed during the current study are available from the corresponding author on reasonable request.

\section{Competing interests}

The authors declare that they have no competing interests

\section{Funding}

This study was funded by FUJIFILM Wako Pure Chemical Corporation. The sponsor had no control over the design, implementation, interpretation of results, writing, or publication of this work.

\section{Authors' contributions}

H.O and J.F designed the study and wrote the initial draft of the manuscript. Y.T contributed to analysis and interpretation of data and wrote the main manuscript text and prepared figures 1-2 and tables. N.I is a statistician who contributed to analysis and interpretation of the data. K.M is a doctor who conceived the concept of this study (took over this study from FUJIFILM Wako Pure Chemical Corporation), and helped us interview the patients. K.N, S.Y, Y.H, A.I, T.Y, T.H, and T.T have contributed to data collection and interpretation, and critically reviewed the manuscript. All authors approved the final version of the manuscript.

\section{Acknowledgements}

I would like to thank Dr Satomi Haruki and Dr Mizuki Imai, the medical staff of the Cancer Screening Centre for helping us to interview patients for their history of $H$. pylori eradication and obtain patients' consent for participating in this study. We received generous support from Kazumi Kaihara, who is a laboratory technician.

\section{References}

1. Marshall BJ, Warren JR. Unidentified curved bacilli in the stomach of patients with gastritis and peptic ulceration. Lancet 1984;1:1311-5.

2. Uemura N, Okamoto S, Yamamoto S, Matsumura N, Yamaguchi S, et al. Helicobacter pylori infection and the development of gastric cancer. N Engl J Med 2001;345:784-9.

3. International Agency for Research on Cancer. IARC monographs on the evaluation of carcinogenic risks to humans. In; IARC,Schistosomes, liver flukes and Helicobacter pylori Vol. 61. Lyon: IARC; 1994.

p. 177-240.

4. Suzuki $H$, Nishizawa T, Tsugawa $H$, Hibi T. Molecular approaches and modern clinical strategies for the management of Helicobacter pylori infection in Japan. Keio J Med 2012;61:109-19.

5. Nishizawa T, Suzuki H. Mechanisms of Helicobacter pylori antibiotic resistance and molecular testing. Front Mol Biosci 2014;1:19. 
6. Asaka M, Kato M, Takahashi SI, Fukuda Y, Sugiyama T, et al. Guidelines for the management of Helicobacter pylori infection in Japan: 2009 revised edition. Helicobacter 2010;15;1:1-20.

7. Ohara S, Kato M, Saito M, Fukuda S, Kato C, et al. Comparison between a new 13 C-urea breath test, using a film-coated tablet, and the conventional $13 \mathrm{C}$-urea breath test for the detection of Helicobacter pylori infection. J Gastroenterol 2004;39;7:621-8.

8. Kimura K, Takemoto T. An endoscopic recognition of the atrophic border and its significance in chronic gastritis. Endoscopy 1969;1:87-97.

9. Kato M, Inoue K, Murakami K, Kamada T, Haruma K. Kyoto Classification of Gastritis. Tokyo: Nihon Medical Centre 2017:97-110.

10. Kita M, Yokota K, Okada H, Take S, Takenaka R, et al. The genetic diversity of Helicobacter pylori virulence genes is not associated with gastric atrophy progression. Acta Medica Okayama 2013;67;2:93-8.

11. Masayuki I, Susumu O, Yoshikatsu I, Yuko K. Analysis of the gray-zone cutoff values of new serum Helicobacter pylori antibody kits using latex immunoassay. Japanese Journal of Gastroenterology. 2017;114:1968-77. [Article in Japanese]

12. lino C, Shimoyama T, Oyama T, Chiba D, Umeda T, et al. Evaluation of the appropriate cut-off value for serological diagnosis of Helicobacter pylori infection by comparison with a stool antigen test. Hirosaki Med J 2012;18;63:48-54.

13. Ohara S, Kato M, Asaka M, Toyota T. Studies of ${ }^{13} \mathrm{C}$-urea breath test for diagnosis of Helicobacter pylori infection in Japan. J Gastroenterol 1998;33;6-13.

\section{Figures}

\section{E-Plate H.pylori antibody II}

by hand and auto

\begin{tabular}{|r}
\hline \\
\hline \\
\hline \\
\hline \\
\hline
\end{tabular}

0 min Add 10 $\mu \mathrm{L}$ sample and $100 \mu \mathrm{L}$ Buffer to each well

Mix well 5 minutes

-10 min Incubate 25 minutes at $15 \sim 30^{\circ} \mathrm{C}$

$20 \mathrm{~min}$

- 30 min Aspirate and wash 3 times

30 min Add $100 \mu \mathrm{L}$ Enzyme labeled anti-human IgG antibody

- $40 \mathrm{~min}$ Incubate 30 minutes at $15 \sim 30^{\circ} \mathrm{C}$

- $50 \mathrm{~min}$

- $60 \mathrm{~min}$ Aspirate and wash 3 times

- 70 min Add $100 \mu \mathrm{L}$ Substrate solution

Incubate 15 minutes at $25^{\circ} \mathrm{C}$

- $80 \mathrm{~min}$

- $90 \mathrm{~min} \begin{aligned} & \text { Add } 100 \mu \mathrm{L} \text { Stop solution } \\ & \text { Read at 492nm immediately }\end{aligned}$

\begin{tabular}{l|l} 
L type wako H.pylori antibody & by auto \\
\hline $\begin{array}{l}\text { Add } 5 \mu \mathrm{L} \text { Sample and } 150 \mu \mathrm{L} R 1 \\
\text { Incubate } 5 \text { minutes at } 37^{\circ} \mathrm{C}\end{array}$ & by auto \\
Add $50 \mu \mathrm{L} 2$ & (ten minutes) \\
Incubate 5 minutes at $37^{\circ} \mathrm{C}$ & \\
Caluculate by automatically &
\end{tabular}

by auto

(87 minutes)

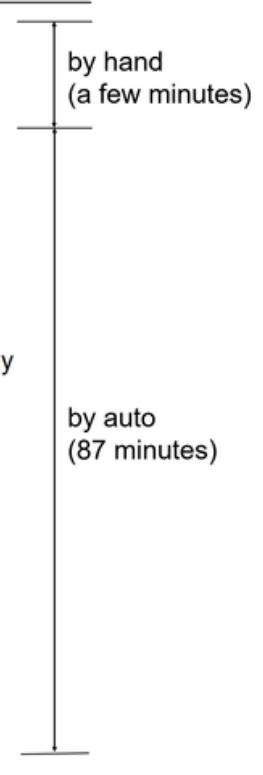

Figure 1

Page $14 / 17$ 


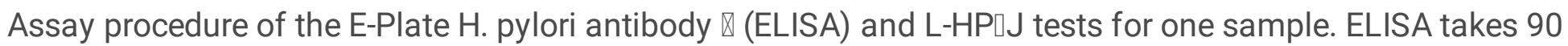
min "by hand and auto" per plate (1-96 samples). In contrast, a biochemical autoanalyser ( $\mathrm{L}$ type Wako $\mathrm{H}$. pylori antibody) requires 10 min for each sample. ELISA, Enzyme-Linked Immunosorbent Assay; L-HP·J, Ltype Wako Helicobacter pylori antibody J.

E-Plate H.pylori antibody II

by hand and auto

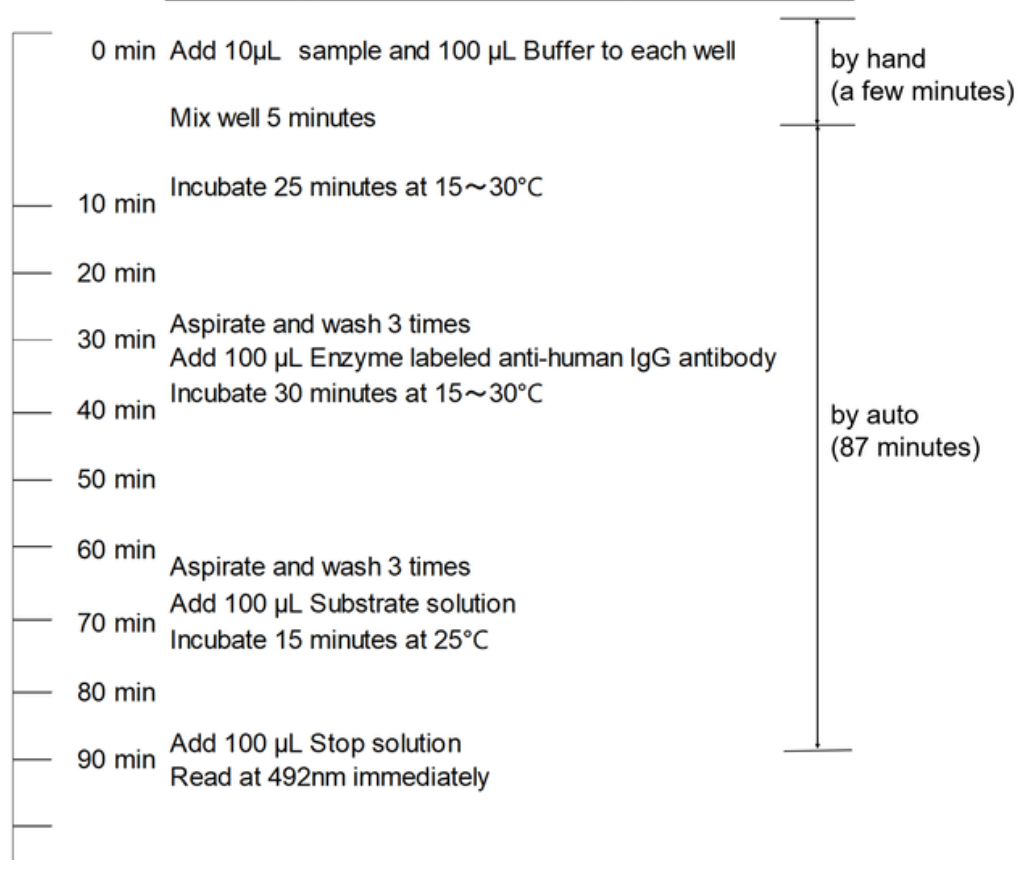

\begin{tabular}{l|l} 
L type wako H.pylori antibody & by auto \\
\hline $\begin{array}{l}\text { Add } 5 \mu \mathrm{L} \text { Sample and } 150 \mu \mathrm{L} \text { R1 } \\
\text { Incubate } 5 \text { minutes at } 37^{\circ} \mathrm{C}\end{array}$ \\
$\begin{array}{l}\text { Add } 50 \mu \mathrm{L} \mathrm{R} 2 \\
\text { Incubate } 5 \text { minutes at } 37^{\circ} \mathrm{C}\end{array}$ \\
$\begin{array}{l}\text { Caluculate by automatically } \\
\text { (ten minutes) }\end{array}$
\end{tabular}

\section{Figure 1}

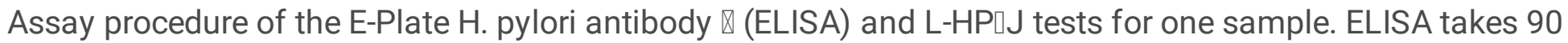
min "by hand and auto" per plate (1-96 samples). In contrast, a biochemical autoanalyser ( $L$ type Wako $\mathrm{H}$. pylori antibody) requires $10 \mathrm{~min}$ for each sample. ELISA, Enzyme-Linked Immunosorbent Assay; L-HP.J, Ltype Wako Helicobacter pylori antibody J. 

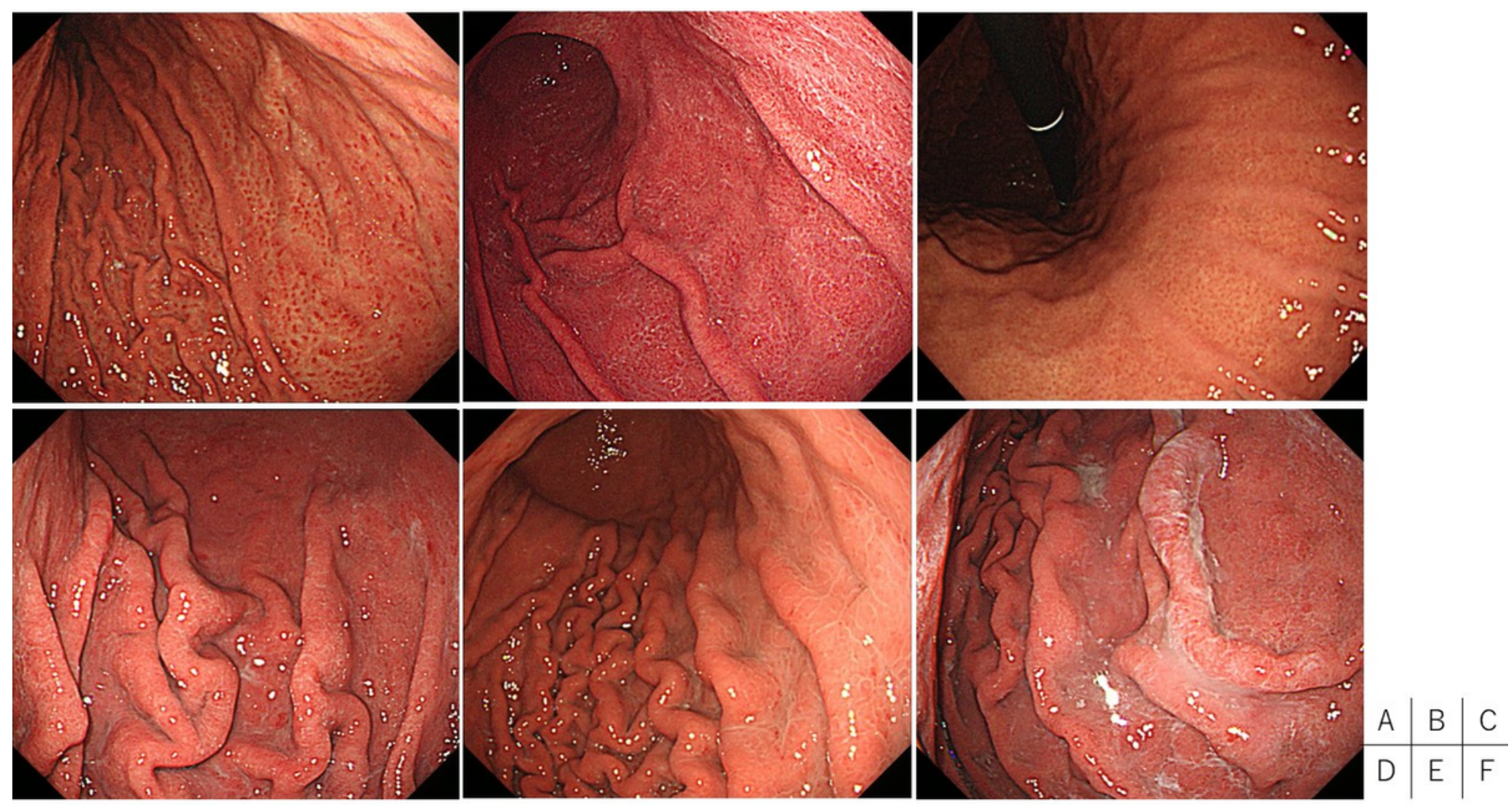

\section{Figure 2}

Endoscopic findings that suggest active gastritis. (A) Spotty redness: multiple tiny reddish spots in the fundic gland region. Spotty redness is usually observed in the upper region of the stomach. (B) Diffuse redness: uniform redness involving the entire mucosa of the fundic gland region. (C) Disappearance of the regular arrangement of collecting venules: starfish-like red spots in a regular arrangement visible through the mucosal surface in the fundic gland region have disappeared due to H. pylori-induced inflammation. (D) Enlarged/tortuous folds. (E) mucosal swelling. (F) sticky mucus. 

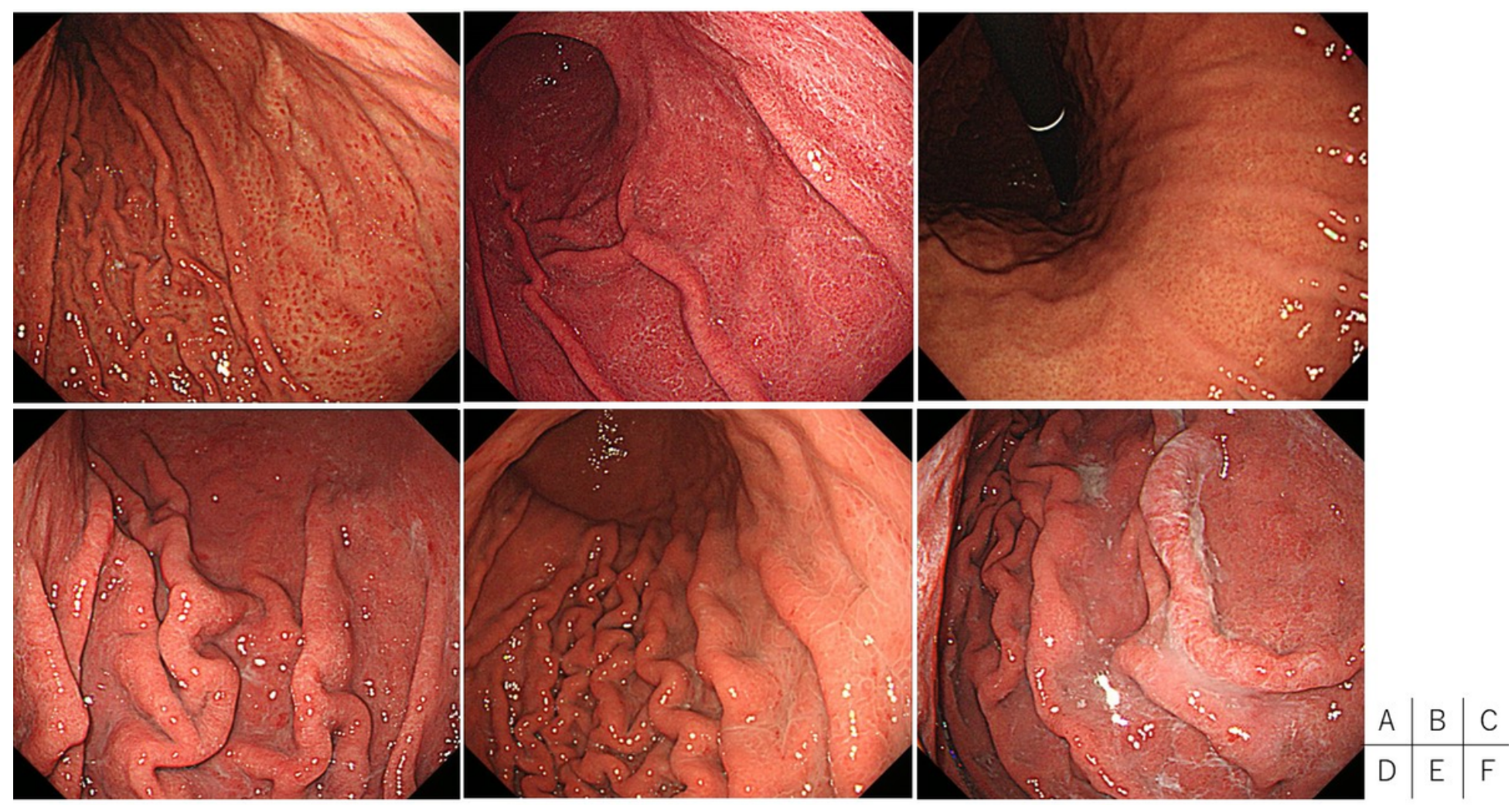

Figure 2

Endoscopic findings that suggest active gastritis. (A) Spotty redness: multiple tiny reddish spots in the fundic gland region. Spotty redness is usually observed in the upper region of the stomach. (B) Diffuse redness: uniform redness involving the entire mucosa of the fundic gland region. (C) Disappearance of the regular arrangement of collecting venules: starfish-like red spots in a regular arrangement visible through the mucosal surface in the fundic gland region have disappeared due to H. pylori-induced inflammation. (D) Enlarged/tortuous folds. (E) mucosal swelling. (F) sticky mucus. 